\title{
Tracer and timescale methods for understanding complex geophysical and environmental fluid flows
}

\author{
Eric Deleersnijder • Fabien Cornaton • \\ Thomas W. N. Haine • Marnik Vanclooster • \\ Darryn W. Waugh
}

Received: 15 December 2009 / Accepted: 16 December 2009 / Published online: 7 January 2010

(C) Springer Science+Business Media B.V. 2010

Understanding advective-diffusive transport of trace constituents in natural fluid flows is an important challenge in Earth and environmental sciences with many diverse applications, including simulating the fate of contaminants, inferring the location of their source, and model assessment (e.g., [7,21]). Eulerian and Lagrangian methods are routinely used, including novel representations of mixing processes that resort to the so-called fractional-order diffusion. Moreover, geophysical and environmental fluid-flow models routinely produce huge amounts of output, and to make sense of these results sophisticated interpretation methods are required. Among these methods, an approach that is becoming progressively more popular consists in using real, or hypothetical, tracers to tag fluid masses and estimate associated timescales, such as age, residence time, and transit time. These timescales lead to very useful diagnoses that are increasingly applied in interdisciplinary environmental studies (e.g. [2,5]).

This special issue presents a number of studies that are relevant to the above-mentioned field of research. Groundwater, soil water, riverine, estuarine, marine and ocean flows are considered, as well as the transport of sinking particles in water.

E. Deleersnijder $(\varangle)$

Centre for Systems Engineering and Applied Mechanics (CESAME),

Université catholique de Louvain (UCL), Louvain-la-Neuve, Belgium

e-mail: eric.deleersnijder@uclouvain.be

F. Cornaton

Centre of Hydrogeology, Université de Neuchâtel, Neuchâtel, Switzerland

e-mail: fabien.cornaton@unine.ch

T. W. N. Haine $\cdot$ D. W. Waugh

Department of Earth and Planetary Sciences, Johns Hopkins University, Baltimore, USA

e-mail: thomas.haine@jhu.edu

D. W. Waugh

e-mail:waugh@jhu.edu

M. Vanclooster

Department of Environmental Sciences and Land Use Planning, Université catholique de Louvain (UCL),

Louvain-la-Neuve, Belgium

e-mail: marnik.vanclooster@uclouvain.be 
The article of Hanert [12] presents a family of non-local dispersion models that do not assume Brownian motion but rather assume the random displacements of the particles follow a heavy-tail Levy distribution, which allows for large displacements. It is seen that such a hypothesis leads to fractional-order Eulerian transport models, which cannot be discretised efficiently by standard finite-difference and finite-element methods. Instead, non-local numerical methods, like the spectral-element method, are shown to be better suited to solve that equation.

Gräwe and Wolff [9] present a Lagrangian particle-tracking model to simulate short-term suspended particulate matter transport. It is well known that Lagrangian transport schemes are quite attractive for such applications, mostly because they give a straightforward physical interpretation of the processes involved and are well suited to massive parallel computers. The authors present three different tracking schemes and apply them to a realistic winter storm event. They show that particle-tracking schemes can accurately reproduce the dynamics provided that the divergence of the diffusivity tensor is handled carefully.

The paper of Ababou et al. [1] deals with the problem of identifying contaminant sources given certain solute distributions in environmental flows. The authors present a novel, stable, and convergent particle-tracking scheme (RAW: Reverse Anti-diffusive Walk) able to back-diffuse concentration plumes, that is, diffusion backwards-in-time. This study is an interesting, theoretical exploration that extends the authors' previous works on Lagrangian particle methods to the challenging task of back-tracking a diffusion process in time in order to identify pollution sources. As stated by the authors the proposed numerical schemes are limited to known isotropic diffusion and, therefore, still need to be generalized to tackle realworld multiple-source identification problems involving heterogeneous transport parameters.

Tosco and Sethi [20] present a comparison between two very different computational approaches for the delineation of well-head protection zone areas in porous media, an important practical problem in hydrogeology. Deterministic well-capture zones (purely advective) are defined using the widely used reverse particle-tracking approach. Probabilistic wellcapture zones (advective and dispersive) are estimated using a backward probability model derived from the adjoint of the advection-dispersion equation. Both types of capture zone areas are compared using simplified synthetic 2-D aquifer configurations. They can display important differences in shape and extent, thus showing the importance of including the dispersion/diffusion phenomena in calculations addressing the delineation of well-head protection zones.

In Lemieux and Sudicky [14], the concept of groundwater age is applied to study the impact of the Wisconsinian glaciation on the Canadian continental groundwater flow systems. Large-scale coupled surface-subsurface water flow and glaciation solutions originating from the authors' previous works are exploited by solving for the transient evolution of groundwater mean age. The authors show that mean age in regions affected by the ice sheet advance and retreat is younger than it was at the last interglacial period. This result also holds for frozen groundwater in the permafrost area, which suggests that significant parts of this water is of glacial origin. Estimated ages (up to $42 \mathrm{Myr}$ ) are in good agreement with the observations made in the Great Lake region (up to $50 \mathrm{kyr}$ ). Meltwater penetration-depth estimates (up to $3 \mathrm{~km}$ ) are also in good agreement with the observations made in the Illinois, Michigan and Williston basins, and in the Canadian Shield. This paper is important as a means of understanding the large-scale impact of an ice sheet on groundwater systems.

In Darracq et al. [3], the distributions of advective solute travel times through hydrological catchments is studied for two hydrological catchments in Sweden. The travel-time distributions are essential for understanding how local water flow and solute transport and attenuation processes affect the catchment-scale transport of solute, for instance, with regard to 
biogeochemical cycling, contamination persistence, and contamination load. Results show that in these hydrological catchments travel times are largely determined by the groundwater component and hence the physical properties describing flow through the subsurface groundwater body such as the hydraulic gradient, porosity, and hydraulic conductivity. Different hypotheses on the spatial variability of these properties have a considerable effect on catchment travel time.

In Mattern and Vanclooster [16], the authors estimate the travel time of percolation water through a deep vadose zone of the Brusselian aquifer by combining a transfer-function model and a physically-based model, and considering time-variable percolation rates. A log-normal probability-density function is chosen for the transfer-function model, in accordance with a porous medium exhibiting a log-normal distribution of pore velocities. The Hydrus-1D model is applied to numerically solve for the 1-D Richards and advective-dispersive solute transport equations. The important discrepancies between the results from the two types of models are explained by the conceptual differences between the two modelling approaches and the uncertainty associated to the parameterization of the physically-based flow and transport models.

Gualtieri [10] examines the values of the transverse diffusion coefficient for depth-integrated simulations of tracer transport in river flows. Numerical results obtained by means of the classical $k-\varepsilon$ closure scheme compare rather favourably with experimental data. This finding is of importance for a wide range of tracer transport simulations as well as for estimating the associated diagnostic timescales.

In de Brauwere and Deleersnijder [4], the concept of residence time is seen to be very efficient to assess depth-integrated models of the fate of decaying and sinking particles. A comprehensive sensitivity analysis is carried out with, and without, stratification.

Seeking inspiration in Mercier and Delhez [17], Gong and Shen [8] estimate the age of sediment in the water column and at the seabed in an estuary, where it helps diagnose transport of material adsorbed on sediment particles. Not surprisingly, the magnitude of the settling velocity and the critical shear stress are seen to impact critically the timescales obtained. These timescales are useful for a range of problems, including the prediction of eutrophication and the fate of contaminants.

Huang et al. [13] estimate water ages in an estuary from the travel-time median of dye released at the entrance of the estuary. At several locations in the estuary this age is seen to be well-correlated with an empirical power law of the river flow. It is underscored that water age is an important indicator of estuarine water quality and, hence, is important in ecosystem assessment.

Orre et al. [18] discuss the transport of ${ }^{129} \mathrm{I}$ in the northern North Atlantic Ocean. In simulations using a global ocean circulation model, the authors find reasonable agreement with field measurements of the isotope. They then study the transport characteristics of their model flow using transit-time distributions, and discuss propagation of other passive tracer anomalies through the sub-polar Atlantic and Nordic Seas.

In Terenzi and Hall [19], new solutions are derived for one-dimensional advection-dilution and advection-diffusion models with non-constant coefficients. These solutions are used to compare tracer fluctuations due to temporal variations in the transport with those due to temporal variations in the boundary conditions. When applied to CFC observations in the North Atlantic Deep Western Boundary Current (DWBC) it is shown that fluctuations resulting from boundary conditions dominate near the source region, while transport fluctuations acting on background tracer gradients dominate away from the source. This contrasts a common view that fluctuations in tracers, such as CFCs, temperature, and salinity, in the subtropical and tropical DWBC are propagated signals from the northern formation region. 
Dutay et al. [6] gauge the impact of geothermal heating of the sea floor and abyssal vertical mixing on an ocean general circulation model, evaluating their results with ${ }^{3} \mathrm{He}$ data. They find that in order to produce realistic ${ }^{3} \mathrm{He}$ simulations, both geothermal heating and enhanced vertical mixing are necessary.

Maltrud et al. [15] present results from simulations of age-related passive tracers in a $1 / 10^{\circ}$ resolution global ocean model. This is the first time age tracers have been analyzed in a global model that resolves eddying motions. A century-long simulation is performed including five global Boundary Impulse Response functions (BIRs), which are used to characterize the related Transit Time Distribution functions (TTDs), and hence the variability in water-mass ventilation timescale in the presence of eddies. The authors show that variability is largest at short timescales, but at longer timescales there is a remarkable conformity between the ensemble of BIR realisations. Furthermore, variations in the BIR first moment over transit time compared to the corresponding ensemble average are typically only a few percent. Invoking the study of Haine et al. [11], these results imply that only a few BIR realisations may be necessary for a reasonable estimate of the TTD.

All of these papers testify to the wide range of applications and the usefulness of tracer and timescale methods. Clearly, this field of research is alive and well. The field has not yet reached full maturity, however, and can be confusing to the newcomer. In particular, similar concepts and equations are sometimes given different names by different authors - even for flows in the same medium. For oceanography alone, Wunsch [22] deplored this situation back in 2002 and, unfortunately, there has been no sign of improvement since then. Attempts towards unification should be made, which, hopefully, would lead to a coherent set of definitions, equations and notations that would be applicable to most, if not all, geophysical and environmental fluid flow problems.

The guest-editors are indebted to the authors and anonymous reviewers for their contributions to this special issue. Eric Deleersnijder is a research associate with the Belgian Fund for Scientific Research (F.R.S.-FNRS). His work was performed under the auspices of the Interuniversity Attraction Pole TIMOTHY (www.climate.be/timothy), which is funded by the Belgian Science Policy office (BELSPO) under contract IAP6.13.

\section{References}

1. Ababou R, Bagtzoglou AC, Mallet A (this issue) Anti-diffusion and source identification with the 'RAW' scheme: a particle-based censored random walk. Environm Fluid Mech

2. Cornaton F, Perrochet P (2006) Groundwater age, life expectancy and transit time distributions in advective-dispersive systems: 1 Generalized reservoir theory. Adv Water Resour 29:1267-1291

3. Darracq A, Destouni G, Persson K, Prieto C, Jarsjö J (this issue) Quantification of advective solute travel times and mass transport through hydrological catchments. Environ Fluid Mech

4. de Brauwere A, Deleersnijder E (this issue) Assessing the parameterisation of the settling flux in a depthintegrated model of the fate of decaying and sinking particles, with application to fecal bacteria in the Scheldt Estuary. Environ Fluid Mech

5. Deleersnijder E, Delhez EJM (eds) (2007) Timescale- and tracer-based methods for understanding the results of complex marine models. Estuar Coast Shelf Sci (Special Issue) 74:585-776

6. Dutay J-C, Emile-Geay J, Iudicone D, Jean-Baptiste P, Madec G, Carouge C (this issue) Helium isotopic constraints on simulated ocean circulations-implications for abyssal theories. Environ Fluid Mech

7. England MH, Maier-Reimer E (2001) Using chemical tracers to assess ocean models. Rev Geophys 39:29-70

8. Gong W, Shen $\mathbf{J}$ (this issue) A model diagnostic study of age of river-borne sediment transport in the tidal York River Estuary. Environ Fluid Mech

9. Gräwe U, Wolff J-O (this issue) Suspended particulate matter dynamics in a particle framework. Environ Fluid Mech 
10. Gualtieri C (this issue) RANS-based simulation of transverse turbulent mixing in a 2D geometry. Environ Fluid Mech

11. Haine TWN, Zhang H, Waugh DW, Holzer M (2008) On transit-time distributions in unsteady circulation models. Ocean Model 21:35-45

12. Hanert $\mathrm{E}$ (this issue) A comparison of three Eulerian numerical methods for fractional-order transport models. Environ Fluid Mech

13. Huang W, Liu X, Chen X, Flannery MS (this issue) Estimating river flow effects on water ages by hydrodynamic modeling in Little Manatee River estuary, Florida, USA. Environ Fluid Mech

14. Lemieux J-M, Sudicky EA (this issue) Simulation of groundwater age evolution during the Wisconsinian glaciation over the Canadian landscape. Environ Fluid Mech

15. Maltrud M, Bryan F, Peacock S (this issue) Boundary impulse response functions in a century-long eddying global ocean simulation. Environ Fluid Mech

16. Mattern S, Vanclooster M (this issue) Estimating travel time of recharge water through a deep vadose zone using a transfer function model. Environ Fluid Mech

17. Mercier C, Delhez EJM (2007) Diagnosis of sediment transport in the Belgian Coastal Zone. Estuar Coast Shelf Sci 74:670-683

18. Orre S, Smith JN, Alfimov V, Bentsen M (this issue) Simulating transport of and idealised tracers in the northern North Atlantic Ocean. Environ Fluid Mech

19. Terenzi F, Hall TM (this issue) Idealized tracer transport models with time-varying transport: applications to ocean boundary currents. Environ Fluid Mech

20. Tosco T, Sethi R (this issue) Comparison between backward probability and particle tracking methods for the delineation of well head protection areas. Environ Fluid Mech

21. Waugh DW, Hall TM (2002) Age of stratospheric air: theory, observations, and models. Rev Geophys 40(4):1010. doi:10.1029/2000RG000101

22. Wunsch C (2002) Oceanic age and transient tracers: analytical and numerical solutions. J Geophys Res 107:3048. doi:10.1029/2001JC000797 